\title{
Putnam e la critica dell'epistemologia contemporanea: spunti pragmatisti
}

Antonio Lizzadri

\section{(2) OpenEdition}

1 Journals

Edizione digitale

URL: http://journals.openedition.org/ejpap/1716

DOI: 10.4000/ejpap.1716

ISSN: 2036-4091

Editore

Associazione Pragma

\section{Notizia bibliografica digitale}

Antonio Lizzadri, «Putnam e la critica dell'epistemologia contemporanea: spunti pragmatisti », European Journal of Pragmatism and American Philosophy [Online], XI-2 | 2019, Online since 24

December 2019, connection on 16 June 2020. URL : http://journals.openedition.org/ejpap/1716 ; DOI : https://doi.org/10.4000/ejpap.1716

\section{Questo documento è stato generato automaticamente il 16 giugno 2020}

\section{c) (i) $\Theta$}

Author retains copyright and grants the European Journal of Pragmatism and American Philosophy right of first publication with the work simultaneously licensed under a Creative Commons AttributionNonCommercial-NoDerivatives 4.0 International License. 


\title{
Putnam e la critica dell'epistemologia contemporanea: spunti pragmatisti
}

\author{
Antonio Lizzadri
}

\section{NOTE DELL'AUTORE}

Desidero ringraziare i revisori anonimi di questo articolo, le cui osservazioni e indicazioni hanno costituito un'occasione proficua di confronto e approfondimento, che mi hanno consentito di migliorare il testo.

\section{Introduzione}

Tra il pragmatismo e la filosofia di Hilary Putnam sussiste una proficua complementarietà teoretica: le rispettive categorie concettuali costituiscono, infatti, un privilegiato e reciproco punto d'osservazione. D'altra parte, Putnam non è stato un mero fruitore passeggero e passivo della tradizione pragmatista, ma ha contribuito a riattualizzarne le istanze sin dagli inizi del suo percorso intellettuale, configurando così la peculiarità delle sue stesse posizioni, sebbene non desiderasse essere etichettato come neopragmatista e, in generale, con nessun tipo di "ismo."

Il proposito di esplorare con più attenzione gli aspetti pragmatisti del pensiero di Putnam sembra dunque avere almeno due motivi di interesse storico-teoretico: innanzitutto, la possibilità di recuperare un criterio interpretativo unitario per l'intricata rete di questioni e soluzioni proposte dall'autore nell'arco di molti decenni laddove l'avvicinamento al pragmatismo, al contrario, viene solitamente considerato un elemento di rottura e discontinuità - nonché la possibilità di rileggere in maniera sintetica l'evoluzione dell'epistemologia contemporanea. Putnam, infatti, è stato opportunamente definito come "la storia della filosofia recente in compendio," 
(Passmore 1988: 87) in quanto si è confrontato con le principali tradizioni epistemologiche, senza limitarsi a coltivarne il ricordo, ma impegnandosi a operare una sintesi, in grado di far fronte in modo attuale alla complessità del dibattito.

In primo luogo, occorre allora evidenziare che l'assunzione matura e consapevole del pragmatismo a partire da Realism and Reason ${ }^{2}$ e Reason, Truth, and History ${ }^{3}$ era stata a ben vedere preparata già nelle ricerche precedenti: i Philosophical Papers del 1975, infatti, lungi dal rappresentare l'esito e il compimento della sua formazione neopositivista, rappresentano piuttosto una critica sistematica di quella visione riduzionista e scientista della realtà, ${ }^{4}$ proprio in nome delle tesi più significative del pragmatismo stesso, tra cui il primato della prassi come criterio di verità della ricerca scientifica e filosofica, il primato del linguaggio ordinario sui linguaggi formali, il rilievo epistemologico della soggettività e dell'esperienza vissuta, l'unità di metodologia e scienze umane, ecc.

Verificheremo l'effettività di tali istanze del pragmatismo nella critica di Putnam ai principali paradigmi epistemologici del secolo scorso. Presenteremo innanzitutto la critica al verificazionismo neopositivista, la cui distinzione tra linguaggio osservativo e linguaggio teorico risulterà inadeguata a fronte del riconoscimento del primato del linguaggio ordinario rispetto ai linguaggi formali. Una maggior attenzione alla prassi risulterà decisiva anche per avvedersi dei limiti del falsificazionismo popperiano, che, assolutizzando l'aspetto predittivo delle teorie scientifiche - peraltro, allo stesso modo del neopositivismo - non riconosce adeguatamente l'importanza del successo esplicativo ed applicativo quale virtù epistemica essenziale al di là del controllo. L'apertura della riflessione epistemologica a fattori extra-teorici come l'ordinaria prassi linguistica e il successo pratico consentirà, infine, di riconoscere piuttosto le affinità di Putnam con gli orientamenti dell'epistemologia post-popperiana. D'altra parte, sarà nuovamente la sensibilità pragmatista del filosofo di Harward a fare la differenza: vedremo come, mentre in Kuhn il rifiuto della tradizionale metodologia della scienza sfoci in una concezione del sapere relativistica, in Putnam, l'attenzione alla pratica scientifica consenta di continuare ad avere fiducia nel progresso, sebbene non vi sia alcuna giustificazione apodittica del ragionamento induttivo.

\section{Critica del verificazionismo}

\subsection{Verificazionismo e solipsismo}

La paradossalità del verificazionismo secondo Putnam dovrebbe emergere dalla semplice constatazione che esso presuppone l'assunto fondamentale della concezione metafisica che si propone di contrastare. Putnam riconosce infatti nel verificazionismo una certa "tendenza idealistica" poiché:

in base ad una spiegazione verificazionista [...] l'unica nozione effettiva di correttezza disponibile per chi pensa è quella di essere verificato. Se questa è la sola nozione di correttezza che si ritiene la mia "mente/cervello" sia capace di impiegare, e se l'abilità di dire che un'affermazione è verificata è la sola abilità che soddisfa il requisito [...] che la mia capacità di distinguere ciò che è vero da ciò che è falso possa "manifestarsi" a livello comportamentale, allora il mio discorso sugli altri è intellegibile solo per me, come mezzo per fare affermazioni che sono o saranno verificate dalle mie esperienze. (Putnam 2012; trad. it. 2012: 105) ${ }^{5}$ 
In altri termini, Putnam sostiene che sia l'idealismo sia il verificazionismo affondino le proprie radici in un medesimo solipsismo metodologico: sebbene l'assolutizzazione dei dati sensoriali sembrerebbe collocare il verificazionismo in una cornice nettamente realista, la radicale soggettività della percezione precluderebbe, a ben vedere, la possibilità di un confronto intersoggettivo degli stessi dati sensoriali, i quali, in definitiva, potrebbero essere verificati solo dal soggetto che li percepisce. D'altra parte, Putnam osserva come si ripeta la vicenda della filosofia moderna, in cui l'assunzione della way of ideas sia nella tradizione empirista sia in quella razionalista era sfociata nell'identificazione berkeleiana tra esse e percipi:

Le ragioni dell'insuccesso dell'idealismo berkeleiano sono le stesse dell'insuccesso del positivismo machiano: Mach dichiara che la sua costruzione del mondo a partire dalle sensazioni ("Empfindungen") è compatibile con il parlare ordinario e scientifico sugli oggetti, ma non dà alcuna dimostrazione di ciò. (Putnam 1973; trad. it. 2004: 231)

E a dire il vero tanto l'idealista quanto l'empirista non possono dimostrare che le loro teorie parlino del mondo, poiché adottando un metodo introspettivo rimangono rinchiusi nel circolo delle proprie "idee," ovvero delle proprie "esperienze":

Oggi il neopositivista non è autorizzato ad accettare la teoria e la pratica scientifica più di quanto lo fosse Berkeley: non vi è nulla nella sua vicenda che lo porti a dover pensare o che la teoria scientifica è vera o che la pratica scientifica tende a scoprire la verità. In un certo senso ciò è immediato. Il positivista non afferma che la teoria scientifica è "vera," in nessun senso transteorico di "vero"; le sole nozioni transteoriche che egli possiede sono del tipo "dà luogo a una predizione riuscita" e "è semplice." Al pari del seguace di Berkeley, egli deve ripiegare sulla posizione che la teoria scientifica è utile piuttosto che vera. (Ibid.: 233)

Tuttavia, Putnam riconosce quantomeno all'interno della tradizione neoempirista un nobile tentativo di superamento dell'introspezione quale fondamento della conoscenza. Rudolf Carnap, infatti, nella Logische Syntax der Sprach ${ }^{6}$ avrebbe cercato di superare le nozioni introspettive di oggetto materiale tipiche dell'empirismo come "fasci di sensazioni" (Mill) o come "costrutti logici a partire dai dati sensoriali" (Ayer), prendendo in considerazione le relazioni teoriche tra sistemi di enunciati.

Carnap, nel pieno spirito del nuovo empirismo, ha intuito come lo studio del linguaggio avrebbe potuto offrire un punto d'appoggio per sottrarsi allo sprofondamento soggettivistico nel "pantano dell'idealismo," cercando, più precisamente, di dimostrare come per ogni enunciato introspettivo di senso comune su oggetti materiali vi sarebbe stato un enunciato corrispondente su dati sensoriali. Un linguaggio di dati sensoriali avrebbe infatti eliminato le nozioni metafisiche di cui è contaminato il discorso ordinario sugli oggetti materiali (comunemente intesi appunto come cose, res), offrendo peraltro in questo modo un fondamento linguistico inconcusso per la costruzione delle teorie scientifiche. ${ }^{8}$

Con Carnap - sostiene Putnam - si assiste dunque al passaggio dal "fenomenismo ontologico" dell'empirismo tradizionale, nel quale la tesi secondo cui gli oggetti materiali sono fasci di sensazioni si riduce ad una petitio principii, essendo - come abbiamo visto - indimostrabile a motivo del solipsismo metodologico presupposto, al "fenomenismo linguistico," che si è invece impegnato a tradurre gli "enunciati cosali" in enunciati di dati sensoriali mediante una traduzione $T$ che ne conservasse il valore di verità e il grado di conferma:

È così nata una nuova disciplina, che ha preso il nome di logica della scienza. Ed è così morto il fenomenismo! Infatti, benché Carnap (e molti altri filosofi) si siano mossi 
pieni di fiducia, trent'anni di ricerca nel campo della logica hanno stabilito che non esiste nessuna applicazione $T$ con le proprietà desiderate (nessuno sa come dimostrare una cosa del genere senza considerare tutte le applicazioni possibili di $T$, il che è evidentemente irrealizzabile), ma che tutti i tentativi di costruire una tale applicazione si risolvono in completi fallimenti. (Putnam 1975; trad. it. 2004: 40) ${ }^{9}$

Fallito il progetto di traduzione fenomenista, secondo Putnam i neopositivisti avrebbero tentato due strade alternative: l'impossibilità di completare l'interpretazione del linguaggio scientifico attraverso un linguaggio di dati sensoriali comportò innanzitutto la necessità di considerare le teorie scientifiche solo come parzialmente interpretate, e in secondo luogo, la necessità di evitare qualsiasi compromissione ontologica nei confronti dei termini teorici restii alla traduzione, rinunciando definitivamente al valore semantico delle teorie scientifiche, ridotte così a puri e semplici calcoli di predizioni precise. ${ }^{10}$ Sfortunatamente però, secondo Putnam, anche la concezione delle teorie scientifiche come calcoli parzialmente interpretati non è sostenibile.

\subsection{L'interpretazione parziale e la dicotomia osservativo-teorico}

Quando si afferma che le teorie scientifiche sono "parzialmente interpretate," si intende dire che solo i termini osservativi sono interpretati direttamente, mentre i termini teorici riceverebbero un significato come conseguenza indiretta della prima interpretazione auto-evidente. Il concetto di interpretazione parziale presuppone dunque che il vocabolario non logico della scienza si divida in due parti: da un lato vi sarebbero i termini osservativi che si applicano agli oggetti pubblicamente osservabili e alle loro qualità (ad esempio "rosso," "essere in contatto," "bacchetta," ecc.) dall'altro, i termini teorici corrispondenti invece ad oggetti e qualità non osservabili ("elettrone," "carica," "sogno," “gene," ecc.). ${ }^{11}$

La precarietà della distinzione è già inscritta nella "storia piuttosto curiosa" della stessa nozione di interpretazione parziale: secondo Putnam, infatti, la nozione sarebbe avvolta da un "alone tecnico"12 che indurrebbe a considerarne la definizione troppo nota all'interno della logica matematica perché ci sia bisogno di ripeterla:

Purtroppo le cose non stanno così! Carnap, infatti, introdusse il termine, senza definirlo, in un paragrafo della sua monografia (Carnap, 1939). Egli asserì, senza dare spiegazioni, che interpretare i termini osservativi di un calcolo significa automaticamente "interpretare in modo parziale" i primitivi teorici. Il termine è stato successivamente usato da Carnap e da altri autori, ma senza ulteriori spiegazioni. (Ibid.: 243) ${ }^{13}$

È allora lo stesso Putnam che si propone di definire esplicitamente la nozione, esplorando se e in che modo sia possibile che l'interpretazione dei termini osservativi di un calcolo costituisca ipso facto l'interpretazione dei primitivi teorici.

Innanzitutto, è opportuno premettere che, per un verificazionista come Carnap, l'interpretazione di un termine osservativo $P$ è immediata poiché consiste semplicemente nello specificare una procedura di verificazione e refutazione dell'appartenenza alla sua estensione:

Se $\bar{a}$ è una costante individuale che designa un individuo $a[\ldots]$, ed è possibile verificare $P(\bar{a})$, allora l'individuo a è nell'estensione del termine $P$; se $P(\bar{a})$ è refutabile allora $a$ è nell'estensione di $\sim P$, la negazione di $P$; e se le procedure di controllo esistenti non si applicano ad $a$ (ad esempio se $a$ non soddisfa le condizioni 
antecedenti specificate nelle procedure di controllo), allora è indefinito se a è nell'estensione di P oppure no. (Putnam 1962; trad. it. 1993: 242)

A questo punto, per il verificazionista tale nozione può essere estesa in modo immediato all'interpretazione dei primitivi teorici di una teoria nel senso che

se dai postulati e dalle definizioni della teoria e/o dall'insieme di tutti gli enunciati osservativi veri segue $P(\bar{a})$, allora $a$ è nell'estensione di $P$; se dai postulati e dalle definizioni della teoria e/o dall'insieme di tutti gli enunciati osservativi veri segue $\sim P(\bar{a})$, allora $a$ è nell'estensione di $\sim P$; in tutti gli altri casi $P(\bar{a})$ ha un valore di verità indefinito. (Ibid.: 243-4)

Ma è davvero possibile operare una simile estensione con tanta disinvoltura? I limiti connessi all'identificazione del significato dei termini teorici con il metodo di verificazione dei termini osservativi diventano palesi non appena si riflette adeguatamente sui casi di indefinitezza:

in effetti, le nostre condizioni di controllo [...] non possono applicarsi a piccole regioni $X$ situate, ad esempio, all'interno del Sole (o all'interno di molti corpi in molti istanti). Otteniamo pertanto il seguente risultato anomalo: l'affermazione che ci sono atomi di elio nel Sole è vera, ma non è né vero né falso che uno di essi si trovi all'interno di una data piccola sotto-regione $X$ ! Fatti analoghi si verificheranno rispetto ad asserzioni teoriche che riguardano l'immensamente grande; ad esempio, può non essere "né vero né falso" che la curvatura media dello spazio è positiva, o che l'universo è finito. $\mathrm{E}$ ancora una volta scoperte scientifiche del tutto comuni saranno costantemente rappresentate come "stipulazioni linguistiche," "estensioni di significato," e così via. (Ibid.: 246)

Putnam ritiene poi possibile rafforzare il risultato appena conseguito, dimostrando come sia inadeguato definire il significato a partire da procedure di verificazione anche per gli stessi termini osservativi. A tal fine, vengono presi in considerazione $i$ "termini disposizionali puri" che nella definizione carnapiana di Testability and Meaning ${ }^{14}$ corrispondono a quei termini introdotti mediante "enunciati di riduzione". Stando alla concezione verificazionista,

In questo caso l'individuo $a$ è o nell'estensione di $P$ o nell'estensione di $\sim P$, purché l'antecedente di almeno un enunciato di riduzione "che introduce" il termine $P$ sia vero di $a$, e altrimenti è indefinito, che $P(\bar{a})$ sia vera o no. (Putnam 1962; trad. it. 1993: 243)

Per chiarire la questione, Putnam presenta il noto esempio del termine "solubile," supponendo per semplicità che esista un solo test di verifica della solubilità: immergere l'oggetto in acqua. Sulla base della precedente definizione, il verificazionista arriverebbe dunque a sostenere che chiamare solubile un oggetto - supponiamo ora delle zollette di zucchero - che non è mai stato immerso in acqua abbia un valore di verità del tutto indefinito.

Ma come conciliare questa tesi con l'uso ordinario del termine solubile, che si estende ovviamente anche a tutte le zollette che non sono mai state immerse in acqua?

Ciò si deve definire una "stipulazione linguistica." In altre parole, secondo questa concezione, noi diamo al termine "solubile" il nuovo significato di "solubile-nelvecchio-senso-di-zucchero"; e ciò che comunemente diciamo essere una evidenza che le zollette di zucchero non immerse sono solubili, dovrebbe invece essere inteso come una evidenza che il nuovo significato del termine "solubile" è compatibile con l'originale "enunciato di riduzione bilaterale." (Ibid.: 245)

Oltre a tale ricostruzione, dalla quale Putnam riesce già a far emergere bene il carattere macchinoso ed estremamente contro-intuitivo della posizione verificazionista, è possibile individuare al suo interno due limiti ben precisi: da un lato, nel passaggio 
dall'uso ordinario del termine solubile alla definizione rigorosa, in realtà, non si assisterebbe a nessun cambiamento o estensione di significato, dall'altro, non vi sarebbe alcun motivo per rifiutare l'applicazione ordinaria:

Ordinariamente "cambiamento di significato" indica il genere di mutamento che ha avuto, ad esempio, la parola inglese "knave" (che un tempo significava "giovane servitore", spesso autore di furti, e che oggi vuol dire "furfante"; e "estensione del significato" indica il genere di mutamento che ha avuto in Portogallo la parola famiglia ("familia") che ha finito per includere la servitù. Se consideriamo questi tipi di mutamento di significato che dovrebbero essere i soli che servono in una teoria linguistica, è falso affermare che nel caso descritto (quando abbiamo concluso che lo zucchero è solubile) la parola "solubile" abbia subito un cambiamento o una estensione di significato. [...] In ogni caso, non c'è in apparenza alcun motivo per cui non si possa accettare la spiegazione usuale. Ciò che si è sempre inteso dicendo che "la tal cosa è solubile" è, ovviamente, che "se la si immergesse nell'acqua si scioglierebbe"; e il caso può essere descritto in maniera adeguata come una inferenza induttiva che ha portato alla conclusione che tutti questi oggetti (zollette di zucchero, immerse o no) sono solubili. [...] Usualmente si obietta che "non è chiaro che cosa significa" l'espressione: "se fosse immerso in acqua si dissolverebbe"; ma non vi è alcuna evidenza linguistica di questa mancanza di chiarezza. (La si può forse interpretare in altri modi?) (Ibid.: 245-6)

Già da questa prima disamina è rilevabile la sensibilità pragmatista del pensiero di Putnam: al sofisticato formalismo del verificazionismo Putnam oppone infatti le istanze peirceane del "senso comune critico," ${ }^{15}$ secondo cui è l'ordinaria prassi linguistica dei parlanti reali, efficace e funzionale alla vita, a costituire il criterio dell'indagine metodologica, non il contrario. Una metodologia che astraesse dall'esperienza vitale diventerebbe dunque un inutile tecnicismo autoreferenziale.

Putnam ci invita così ad allargare lo sguardo, affrontando la questione non solo dal punto di vista tecnico, ma nella sua veste genuinamente filosofica: ha davvero una consistenza teoretica la distinzione tra termini osservativi e termini teorici? Perché mai si dovrebbe supporre che sia o dovrebbe essere possibile fornire il significato dei termini teorici usando solo termini osservativi? ${ }^{16}$

Putnam predispone la risposta a tali quesiti prestando nuovamente attenzione al nostro effettivo comportamento linguistico, dal quale sarebbe possibile riconoscere come l'ostinazione nel voler eliminare qualsiasi elemento non osservativo nel definiens di un termine teorico sia vana e inopportuna: a ben vedere infatti, nessun lessicografo nella costruzione di un vocabolario si preoccuperebbe della presenza di circolarità nelle proprie definizioni, poiché sa bene che non è la definizione in sé a garantire la comprensione del significato di un termine e che, più in generale, non è la conoscenza del vocabolario a garantire la conoscenza della lingua; semmai, la consultazione di un vocabolario può essere utile e proficua solo nel caso in cui un parlante conosca già la lingua, vale a dire, solo nel momento in cui l'esercizio di un'abilità - l'abilità linguistica appunto - si sia consolidato come habitus stabile. Allora, anche riguardo al significato dei termini teorici, il vero problema non è la correttezza formale della definizione, ma il modo in cui impariamo ad usarli:

i termini teorici si apprendono essenzialmente nel modo in cui si apprende la maggior parte delle parole. A volte ci vengono date delle definizioni lessicali; più spesso imitiamo semplicemente altri parlanti; molte volte uniamo le due cose (ad esempio, ci viene data una definizione lessicale dalla quale ricaviamo un'idea approssimativa dell'uso della parola, e rendiamo poi simile il nostro comportamento linguistico a quello degli altri parlanti, per imitazione). (Putnam 1962; trad. it. 1993: 246) ${ }^{17}$ 
Tra tutte queste eventualità, l'unica certezza è dunque che l'apprendimento del significato di un termine non può essere garantito dall'osservanza di una procedura di verificazione, poiché la componente metodologica si realizza sempre all'interno di una prassi linguistica costituita da elementi empirici di natura psicologia, sociologica, ecc. D'altra parte, secondo Putnam, l'antecedenza logica del linguaggio ordinario sul linguaggio formale non deve essere affatto intesa come mancanza di regolarità $\mathrm{e}$ razionalità nell'apprendimento del significato, poiché è la stessa ricerca scientifica ad averla sempre presupposta. Nella fisica, ad esempio, le grandezze vengono spesso definite tramite delle circonlocuzioni in cui ci si avvale di "figure" tipiche del linguaggio ordinario, quali l'esemplificazione per metafora e analogia, nonché di nozioni qualitative:

si potrebbe definire la "massa" come "quella grandezza fisica che determina la resistenza del corpo all'accelerazione: ad esempio, se un corpo ha massa doppia, sarà doppiamente difficile accelerarlo." Invece di "grandezza fisica" si potrebbe poi dire, nel linguaggio comune, "quella proprietà del corpo," oppure "ciò che nel corpo..."; siffatte nozioni "ad ampio spettro" ricorrono in ogni linguaggio comune. (Putnam 1962; trad. it. 1993: 248; corsivo mio)

E quando nel linguaggio ordinario non sono reperibili soluzioni di continuità con l'uso tecnico di un termine, si osserva la ricorrenza di "asserzioni metalinguistiche" oppure di correlazioni con usi precedenti vicini al linguaggio ordinario, attraverso cui è comunque possibile ricavare un'idea approssimativa del suo significato, che può in seguito essere facilmente precisata esaminando l'intera teoria (cf. ibid.).

Dopo aver constatato come nell'effettiva indagine scientifica vengano inevitabilmente considerati primitivi non solo i termini osservativi e i termini logici ma anche i termini "ad ampio spettro" come "oggetto," "grandezza fisica," ecc., e certe nozioni del linguaggio ordinario imprecise ma utili come "più difficile da accelerare," non dovremmo più nutrire alcuna diffidenza nemmeno rispetto all'introduzione dei termini teorici; e se qualcuno continuasse a dire:

"Pretendo che $\mathrm{i}$ termini teorici vengano introdotti usando soltanto $i$ termini osservativi di Carnap," bisognerebbe rispondere che ciò sembra impossibile. Ma perché mai poi dovrebbe essere possibile? E quale morale filosofica ricavarne? Forse soltanto questa: che è possibile avere un vocabolario teorico ricco come il nostro perché, grazie al cielo, non ci siamo mai trovati nella situazione di avere a disposizione soltanto il vocabolario osservativo di Carnap. (Ibid.: 250)

La critica al verificazionismo appena condotta può apportare alcuni risultati significativi rispetto alle finalità del presente articolo: sebbene fino alla metà degli anni Settanta Putnam abbia sviluppato le questioni epistemologiche ereditate dalla tradizione neopositivista dei suoi maestri attraverso uno stile squisitamente analitico, è tuttavia già possibile intravedere spunti pragmatisti significativi nel rifiuto del solipsismo metodologico, della dicotomia tra linguaggio osservativo e linguaggio teorico, nonché nel riconoscimento del primato del linguaggio ordinario e della prassi linguistica, rispetto a qualsivoglia forma di astratto "deduttivismo" o di "feticismo per il metodo," ${ }^{18}$ che pretenda assurgere a garante imprescindibile della verità. Infatti, il riconoscimento del primato del linguaggio ordinario dimostra piuttosto come i parlanti operino già "nell'ambito della verità", ovvero all'interno di un'interazione effettiva ed efficace col mondo. Di conseguenza, la metodologia può (e deve) avere una funzione propriamente esplorativa di tale interazione e corrispondenza dinamica e asintotica tra parlanti e ambiente, ${ }^{19}$ impegnandosi a riconoscerne le manifestazioni nella prassi senza 
avanzare pretese fondative, giacché sarebbe vano voler fondare ciò che sta a fondamento della conoscenza stessa e che opera come sua condizione di possibilità.

\section{Critica del falsificazionismo}

\subsection{Lo "schema induttivistico" tra verificazionismo e falsificazionismo}

La precarietà della distinzione tra termini osservativi e termini teorici, nonché la conseguente insostenibilità della concezione delle teorie scientifiche come linguaggi parzialmente interpretati hanno inevitabili ricadute sulla tesi complementare, secondo la quale le teorie scientifiche sarebbero meri strumenti di calcolo di previsioni. Tale tesi risulta complementare in quanto il tentativo di ridurre i termini teorici a termini osservativi presuppone, evidentemente, che non si intenda nemmeno assumere un impegno ontologico circa l'esistenza delle entità teoriche da essi designate, sicché, oscurando del tutto il rilievo ontologico delle teorie scientifiche, esse rinunceranno alla loro funzione semantico-descrittiva per assolvere a una funzione meramente sintattica di deduzione e calcolo di previsioni.

Tuttavia, anche quest'ulteriore "forzatura" dell'impalcatura verificazionista è destinata ad infrangersi sulla solidità della reale prassi dell'indagine scientifica:

in moltissimi casi importanti - infatti - le teorie scientifiche non implicano affatto

predizioni. (Putnam 1974; trad. it. 1993: 278)

Il cosiddetto "schema induttivistico," che esplicita i nessi sintattici tra teoria e predizione, non è l'unico modello esplicativo che ricorre nelle scienze, e nemmeno il più importante. Anzi, a ben vedere, una teoria in quanto tale (cioè come puro insieme di leggi) non può garantire alcuna predizione. Si consideri, ad esempio, la teoria della gravitazione universale (GU), costituita dalle tre leggi di Newton e dalla legge secondo cui la forza gravitazionale sussistente tra due corpi è direttamente proporzionale al prodotto delle loro masse e inversamente proporzionale al quadrato della loro distanza. Tale teoria, in se stessa, non può prevedere alcun enunciato di base valido per la verifica, poiché non dice nulla sull'esistenza di altre forze, o di interferenze di altra natura o di ulteriori corpi che potrebbe modificarne il moto. Ed è così che quando si applica la teoria di Newton ad un problema di astronomia - ad esempio, la previsione dell'orbita della Terra - affinché la deduzione sia possibile, è necessario ricorrere ad alcune asserzioni ausiliarie (AA) che, in prima approssimazione, escludano l'esistenza di altri corpi oltre il Sole e la Terra e che prevedano che tali corpi si trovino nel vuoto assoluto e non siano sottoposti ad altre forze se non alle forze gravitazionali mutuamente indotte. La predizione potrà poi certamente essere migliorata, rendendo più realistiche le asserzioni ausiliarie incorporando, ad esempio, altri pianeti nel modello di sistema solare; ma ciò che a Putnam preme sottolineare è che le predizioni non provengono dalla sola teoria, bensì dall'unione della teoria con le AA.

Si potrebbe pensare che la preoccupazione di Putnam sia meramente terminologica, dal momento che basterebbe considerare le asserzioni ausiliarie come parte integrante della teoria per continuare ad adottare tranquillamente lo schema induttivistico senza ulteriori preoccupazioni. Tuttavia,

Non si tratta semplicemente del fatto che gli scienziati non usano il termine

"teoria" per riferirsi alla unione della GU con le AA, bensì che tale uso renderebbe 
oscure alcune profonde questioni metodologiche. [...] Non chiarire la differenza tra le AA e la GU significa non chiarire la differenza tra leggi e asserzioni accidentali, fra asserzioni che lo scienziato si propone di stabilire come vere (le leggi) e asserzioni che egli sa già essere false (le ipotesi estremamente semplificatrici). (Ibid.: 279)

Se - come abbiamo letto - gli scienziati non mancano di riconoscere l'essenzialità della distinzione, sono ancora una volta gli epistemologi ad "oscurare" questioni metodologiche importanti. Questa volta, però, Putnam non prende solo di mira i verificazionisti, ma, con una delle sue audaci interpretazioni storiografiche, assimila questi ultimi ad uno dei loro avversari più emblematici: Karl Popper. Popper, infatti, avrebbe negato proprio la possibilità di verificare le leggi generali sulla base di dati osservativi o sperimentali (che egli chiama "asserzioni-base"), poiché un metodo del genere dovrebbe essere giustificato o come sintetico a priori (ipotesi storicamente non più percorribile in seguito alla scoperta delle geometrie non euclidee e della teoria della relatività) o ricorrendo ad un principio di ordine superiore (ipotesi che condurrebbe inevitabilmente ad un regresso all'infinito).

Popper ovviamente non intende concludere che l'indagine scientifica sia irrazionale poiché fondata su un principio che è impossibile giustificare, ma che questo principio non sia l'induzione. Con ciò Popper non intende nemmeno negare che gli scienziati enuncino leggi generali e che le sottopongano a verifica, ma vuole sostenere che, quando questo avviene, non si stia asserendo la loro verità, e nemmeno la loro probabilità, ma semplicemente che la legge ha superato un controllo. In definitiva, secondo Popper, gli scienziati non sarebbero principalmente impegnati nel verificare leggi, bensì nel falsificarle (cf. ibid.: 274).

Sulla base di questa breve ricostruzione del pensiero di Popper, Putnam osserva innanzitutto che, sebbene le questioni sollevate siano certamente opportune e legittime dal punto di vista logico, la soluzione proposta dal falsificazionismo, presupponendo un atteggiamento di costante sospetto e diffidenza nei confronti delle teorie, è totalmente irrealistica, poiché, a ben vedere, nella prassi abbiamo bisogno di poterci fidare delle nostre idee:

Quando uno scienziato accetta una legge, egli raccomanda agli altri di fare affidamento su di essa - di farvi affidamento, spesso, in contesti pratici. Solo sradicando completamente la scienza dal contesto in cui essa nasce in realtà - il contesto di uomini che tentano di cambiare e controllare il mondo - Popper è in grado di proporre la sua concezione peculiare dell'induzione. Le idee non sono soltanto idee; esse costituiscono una guida per l'azione. Le nostre nozioni di "conoscenza," "probabilità," "certezza," ecc. sono tutte legate a contesti e sono frequentemente adoperate in contesti in cui si pone il problema di come agire: posso avere piena fiducia in una data idea? Devo fare affidamento su di essa a titolo di prova, con una certa cautela? È necessario controllarla? (Ibid.: 275)

Si vede dunque come Putnam ricorra a considerazioni pragmatiste anche nella critica al falsificazionismo che, al pari del verificazionismo, proporrebbe una concezione astratta della conoscenza poiché separata dal contesto pratico entro cui nasce, ovvero "il contesto di uomini che tentano di cambiare e controllare il mondo": analogamente al "socialismo logico" peirceano, anche secondo Putnam "le idee non sono soltanto idee," ma costituiscono una "guida per l'azione." Considerando questa strutturale apertura del conoscere alla prassi, Putnam evidenzia inoltre che le virtù epistemiche delle nostre teorie non potranno essere solo di natura teoretica, ma dovranno essere anche di natura etica, quali la fiducia e l'affidabilità: se non potessimo fidarci delle nostre teorie in contesti pratici, anche le conferme più evidenti e i controlli più severi non avrebbero 
alcuna importanza scientifica. Ritorna dunque anche in Putnam un altro importante elemento pragmatista: la non assolutezza della logica e la sua interdipendenza con altre forme di sapere umanistiche, quali l'etica e l'estetica. Di conseguenza, lungi dall'approvare la concezione scientifica del mondo dell'empirismo logico che sottraeva al dominio della razionalità gli aspetti esistenziali dell'umano, Putnam intende piuttosto riconoscere e ribadire il pieno valore conoscitivo e razionale anche delle credenze morali e religiose derivanti dalle disposizioni cognitive - e non solo emotive della fiducia e della fede. ${ }^{20}$

Passiamo ora alla seconda critica che Putnam rivolge sia al verificazionismo sia al falsificazionismo. Nonostante l'obiettivo polemico fondamentale di Popper sia proprio il principio di induzione del verificazionismo, a ben vedere, anche il falsificazionismo presupporrebbe lo schema induttivistico "teoria-predizione" come unico modello della spiegazione scientifica, rimanendo così anch'esso "impotente" di fronte a tutta una serie di dinamiche strutturali nella scienza, rilevabili solo attraverso il riconoscimento del ruolo fondamentale rivestito dalle asserzioni ausiliarie:

Le spiegazioni "induttivistiche" tipiche della conferma delle teorie scientifiche sono più o meno di questo genere: una teoria implica predizione; se la predizione è falsa, la teoria è falsificata; se un numero sufficientemente alto di predizioni risulta vero, la teoria è confermata. Nonostante tutti i suoi attacchi all'induttivismo, lo schema di Popper non è poi tanto diverso: una teoria implica predizione; se la predizione è falsa, la teoria è falsificata; se un numero sufficientemente alto di predizioni risulta vero, e certe ulteriori condizioni sono soddisfatte, la teoria è corroborata in alto grado. (Putnam 1974; trad. it. 1993: 277; corsivo mio)

Popper aveva previsto che il falsificazionismo avrebbe potuto essere interpretato, contre lui, proprio come una teoria dell'induzione, ed è per questo che vi aggiunse un'importante "condizione antibayesiana"(ibid.): ${ }^{21}$ negando che abbia senso parlare di "probabilità antecedente" di un'ipotesi (antecedente cioè al sussistere o meno della sua predizione), secondo Popper, le predizioni effettuate sulla base del retroterra di conoscenza fornito semplicemente dalla teoria non possono costituirne un controllo; gli unici controlli legittimi sarebbero dunque quelli effettuati sulla base delle predizioni più improbabili rispetto al retroterra di conoscenza, vale a dire i controlli che si contraddistinguono per la loro "severità."

Purtroppo, anche questa condizione aggiuntiva è estremamente lontana dalla reale prassi della ricerca scientifica. Al riguardo, Putnam propone un esempio tratto dalla storia della scienza:

vediamo in che modo fu accettata la GU. Newton in primo luogo ricavò le leggi di Keplero dalla GU e dalle AA: non si trattava di un "controllo" nel senso popperiano, perché già si sapeva che le leggi di Keplero erano vere. In seguito egli mostrò che la GU avrebbe potuto spiegare le maree sulla base dell'attrazione gravitazionale della luna: anche in questo caso non si trattava di un "controllo," in senso popperiano, perché le maree erano già note. Egli impiegò poi molti anni a mostrare che era possibile spiegare, tramite la GU, le piccole perturbazioni delle orbite dei pianeti (le quali erano già note). Ormai l'intero mondo civile aveva accettato - e, in realtà, acclamato - la GU; ma essa non era stata affatto "corroborata" nel senso di Popper! Se cerchiamo un controllo alla Popper della GU - se cerchiamo di ricavare, cioè, una predizione nuova e azzardata rispetto al retroterra di conoscenza - non ne troviamo nemmeno una fino all'esperimento di Cavendish del 1781: all'incirca cento anni dopo che la teoria era stata introdotta! (Ibid.: 291)

Essendo dunque l'appello a "condizioni antibayesiane" inefficace rispetto al tentativo di demarcazione tra corroborazione e induzione, Putnam può così procedere nella critica 
dello "schema induttivistico," per dimostrare quali siano sia nel verificazionismo sia nel falsificazionismo le gravi conseguenze del mancato riconoscimento del ruolo fondamentale delle asserzioni ausiliarie nelle spiegazioni scientifiche.

\subsection{II "successo" oltre le "previsioni"}

Per fare ciò, Putnam rivolge nuovamente l'attenzione all'astronomia, presentando il caso del calcolo dell'orbita di Urano.

Quando intorno al 1846 le predizioni sull'orbita di Urano si rilevarono errate, gli astronomi non pensarono affatto a modificare le leggi di GU, ma rivolsero subito l'attenzione alle asserzioni ausiliarie, e in particolare, a quelle relative al numero dei pianeti. Si trattava infatti dell'“aggiustamento" più semplice, giacché matematicamente parlando - il ripensamento di un mezzo di propagazione della forza che non fosse il vuoto, o il ripensamento dell'influsso di altri generi di forze non gravitazionali, implicando un dominio di alternative indeterminato, avrebbe richiesto di gestire un dominio di previsioni equiprobabili potenzialmente infinito. Invece, con i pianeti, sarebbe bastato procedere ricorsivamente, fintanto che la correzione esatta del loro numero avrebbe consentito di dedurre la previsione corrispondente all'effettiva orbita del pianeta incognito.

Una schematizzazione del problema può facilitarne la comprensione. Dato che il comportamento dell'orbita di Urano si era rivelato incompatibile rispetto alle previsioni della GU, gli astronomi provarono a derivare i nuovi dati sperimentali, a partire da ulteriori asserzioni ausiliarie oltre a quelle già note (siano queste ultime “A1"):

(I) Teoria: GU

AA: (A1)

Ulteriori AA: ?

Explanandum: l'orbita di Urano

Per i motivi di semplicità già esposti, ipotizzarono innanzitutto che oltre a Mercurio, Venere, la Terra, Marte, Saturno e Urano, vi fosse nel sistema solare un solo altro pianeta - sia quest'ulteriore ipotesi "A2" - e sulla base di tale variazione calcolarono l'orbita " $O$ " che il pianeta incognito avrebbe dovuto avere, per poter verificare infine tale predizione:

(II) Teoria: GU

AA: A1, A2

Predizione da verificare: Esiste un pianeta la cui orbita è "O"?

E fu così che nel 1846 Leverrier in Francia e Adams in Inghilterra scoprirono contemporaneamente l'esistenza di un nuovo pianeta: Nettuno, il cui influsso gravitazionale poteva finalmente spiegare le anomalie dell'orbita di Urano (cf. ibid:: 285-7).

Quale insegnamento è possibile trarre dall'intera vicenda? Secondo Putnam, va innanzitutto osservato come lo schema induttivistico "teoria-predizione," esemplificato da (II), intervenga in modo operativo nella spiegazione solo in seguito alla predisposizione di (I), vale a dire, solo dopo aver introdotto tentativamente - e non 
apoditticamente - ulteriori ipotesi ausiliarie. La predizione, dunque, non sarebbe stricto sensu una conseguenza logica, poiché viene dedotta da premesse rispetto alle quali non si ha certezza. E in effetti, l'ipotesi secondo cui vi sarebbe stato un solo pianeta oltre ai sei conosciuti - senza la quale non sarebbe stato possibile derivare la predizione - non era vera: Nettuno non era l'unico pianeta del sistema solare a mancare all'appello; doveva infatti essere ancora scoperto Plutone.

Dobbiamo forse per questo ritenere che Leverrier e Adams si fossero ingannati o che non fossero legittimati ad affermare l'esistenza di Nettuno? Certamente no, saremmo piuttosto indotti a riconoscere come una parte importante dell'attività scientifica non si realizzi attraverso il calcolo di previsioni, e di conseguenza, come la verifica, o corroborazione che dir si voglia, non costituisca l'unico criterio di giustificazione nella scienza. D'altra parte, il fatto stesso che di fronte alle anomalie dell'orbita di Urano non si sia nemmeno pensato di modificare la teoria della gravitazione universale, e che invece si sia da subito intervenuti sulle asserzioni ausiliarie, dimostra come predizioni errate non siano condizione sufficiente per abbandonare una teoria, ma allo stesso tempo, dimostra anche come il suo successo esplicativo sia condizione sufficiente per continuare a mantenerla, pur nella presenza di anomalie. Fintanto che una teoria consente di spiegare "sistemicamente" i fenomeni più significativi all'interno di un particolare paradigma scientifico, tale teoria viene mantenuta, sebbene il suo potere predittivo possa essere stato indebolito da false predizioni. In questa fase della vita di una teoria, la principale attività scientifica non consiste allora nel calcolare previsioni, ma nel trovare le asserzioni ausiliarie necessarie per "far tornare i conti" in virtù del suo reale successo esplicativo.

Se esistono dunque momenti nella scienza - e peraltro nemmeno così "passeggeri" - in cui le predizioni non sono determinanti in vista della giustificazione di una teoria, possiamo finalmente comprendere il difetto fondamentale comune sia al verificazionismo sia al falsificazionismo:

Gli insuccessi non falsificano una teoria, perché un insuccesso non è una falsa predizione ricavata da una teoria unita a fatti noti e sicuri, ma un insuccesso nel trovare qualcosa - in effetti un insuccesso nel trovare una AA. Le teorie, nel periodo della loro permanenza in carica, sono altamente immuni da falsificazioni; la loro carica decade quando compare sulla scena una teoria migliore (o una tecnica esplicativa completamente nuova), non un enunciato di base. E i successi non "confermano" una teoria, una volta che essa è divenuta paradigmatica, perché la teoria non è un'"ipotesi" che abbia bisogno di conferma, ma la base di un'intera tecnica di spiegazione e predizione, e forse anche di una tecnologia. (Ibid.: 288)

In definitiva, la scienza secondo Putnam progredirebbe attraverso la dialettica di due "tendenze conflittuali" e nel contempo "interdipendenti": lo schema "teoria-AA" - (I), per intenderci - rappresenterebbe la "tendenza esplicativa" della ricerca scientifica, in cui una certa teoria viene accettata principalmente in virtù del suo reale successo esplicativo e il maggior sforzo teoretico viene impiegato nella ricerca delle asserzioni ausiliarie che consentano di gestirne le anomalie; quando il "costo di gestione" delle anomalie comincia a diventare insostenibile rispetto al "guadagno esplicativo," e soprattutto, quando inizia a configurarsi un modello teorico alternativo, entra allora in gioco la "tendenza critica" - rappresentata dallo schema (II) "teoria-predizione" - in cui la teoria non viene più data per nota, ma percepita come problematica, e ci si impegna nel metterla alla prova attraverso il controllo delle sue previsioni. 
Non è difficile vedere, infine, come tale conflittualità sia ad un tempo interdipendenza: la soluzione di un problema rappresentato dallo schema (I) deve essere confermata mediante un tipo di controllo della forma dello schema (II), e se la soluzione confermata è una legge generale (e non semplicemente un'asserzione singolare), questa può a sua volta divenire un paradigma proponibile come premessa maggiore di un problema dello schema (I), e così via (Cf. Putnam 1974; trad. it. 1993: 289).

Anche al termine di questa critica del falsificazionismo emergono chiaramente $i$ presupposti pragmatisti dell'argomentazione di Putnam, che ribadisce il primato logico e cronologico della prassi, ovvero del successo esplicativo, applicativo e tecnologico di una teoria, come principio e criterio del progresso scientifico e dell'evoluzione umana in generale:

la prassi è primaria: le idee non sono solo fine a se stesse, né lo è la scelta di quali idee "sottoporre a critica." L'importanza primaria delle idee sta nel fatto che esse guidano la prassi, che strutturano intere forme di vita. Le idee scientifiche guidano la prassi nella scienza, nella tecnologia, e a volte nella vita pubblica e privata. Nella scienza il nostro scopo è il tentativo di scoprire idee corrette: al contrario di ciò che dice Popper, non si tratta di oscurantismo, ma di responsabilità. (Ibid.: 292) 22

\section{Critica dell'epistemologia post-popperiana}

Il ricorso al pragmatismo non risulta "vincente" solo nei confronti del verificazionismo e del falsificazionismo, ma consente a Putnam di superare anche le aporie del terzo momento fondamentale nello sviluppo dell'epistemologia contemporanea, rappresentato da Thomas Kuhn con l'opera Struttura delle rivoluzioni scientifiche.

Putnam riconosce innanzitutto come il dinamismo del progresso scientifico appena delineato corrisponda in qualche modo alle idee centrali di Kuhn di "paradigma," "scienza normale" e "soluzione di rompi capo." Secondo Kuhn, infatti, un "paradigma" è una teoria che dispone di applicazioni "riuscite e straordinarie" - o per usare la terminologia che abbiamo finora adottato - una teoria, il cui successo esplicativo deve essere tale da indurre gli scienziati a emularlo, ricercando ulteriori spiegazioni sulla base dello stesso modello. Come abbiamo letto in precedenza, ciò è accaduto nel momento in cui - introdotta la teoria della gravitazione universale - vennero derivate le leggi di Keplero e spiegato il fenomeno delle maree. Un nuovo paradigma, dunque, è tanto più accolto quanto più è in grado di generare un "campo di ricerca": il campo di ricerca generato dal paradigma newtoniano fu, in primo luogo, l'intero campo della meccanica celeste. Kuhn sostiene inoltre che il paradigma che struttura un intero campo di ricerca sia "altamente immune da falsificazione," poiché nei periodi di "scienza normale," in cui il paradigma è pressoché completamente accettato dalla comunità scientifica, la teoria non riveste il ruolo di una ipotesi da sottoporre a controlli, ma piuttosto "la base di un'intera tecnica di spiegazione," e in particolare della spiegazione delle anomalie attraverso la ricerca di ulteriori asserzioni ausiliarie. Al riguardo, il termine "rompicapo" sembra essere particolarmente efficace poiché evoca l'immagine del tappare un buco, proprio come avviene nella soluzione di un puzzle.

Pur riconoscendo una simile affinità di idee, Putnam ne rivendica l'originaria paternità:

Di recente diversi filosofi hanno iniziato a proporre una concezione piuttosto nuova dell'attività scientifica. Credo di aver anticipato questa concezione dieci anni fa, quando sostenni che certe teorie scientifiche non possono essere confutate sulla 
base di soli esperimenti e osservazioni, ma unicamente tramite teorie alternative. Questa concezione è stata anche anticipata da Hanson (1958), ma viene espressa nel modo più preciso negli scritti di Thomas Kuhn (1962) e Louis Althusser (1965). Entrambi questi filosofi, io credo, commettono degli errori; ma credo anche che la tendenza che essi rappresentano (e che anch'io rappresento, quanto a questo) sia un necessario correttivo al deduttivismo che abbiamo esaminato. (Ibid.: 283) ${ }^{23}$

Il luogo in cui Putnam avrebbe presentato un'inedita concezione dell'attività scientifica è un saggio pubblicato per la prima volta il 22 ottobre 1962 in "The Journal of Philosophy", intitolato It ain't necessarily so. ${ }^{24}$ D'altra parte, anche l'opera di Kuhn The Structure of Scientific Revolutions risale al 1962. La questione interessante non è certamente stabilire a chi spetti il primato, ma provare a capire la peculiarità di ciascuna posizione. A tal fine, prendiamo innanzitutto in considerazione le critiche che Putnam rivolge a Kuhn:

Le asserzioni più controverse di Kuhn riguardano il processo attraverso il quale un nuovo paradigma soppianta un paradigma precedente. Qui egli tende ad essere soggettivista in modo radicale: i dati non possono stabilire la priorità di un paradigma sull'altro perché essi stessi vengono percepiti attraverso le lenti dell'uno e dell'altro paradigma. Il passaggio da un paradigma a un altro richiede un "riorientamento gestaltico." La storia e la metodologia della scienza vengono riscritte ogni volta che avviene un cambiamento di paradigma, pertanto non esistono canoni storici e metodologici "neutrali" a cui richiamarsi. Kuhn sostiene dunque delle concezioni sul significato e sulla verità che hanno carattere relativistico e che sono, a mio avviso, scorrette. (Putnam 1974; trad. it. 1993: 284)

Sebbene Putnam sia convinto, al pari di Khun, che nella giustificazione delle teorie scientifiche il coinvolgimento di elementi extra-metodologici - quali il successo esplicativo - sia determinante, tuttavia, non ritiene che tale relativizzazione della metodologia debba necessariamente comportare una concezione relativistica della conoscenza. In tal senso, lo sforzo di Putnam nel trovare un compromesso virtuoso tra fondazionalismo radicale e relativismo estremo è davvero ammirevole: se da un lato le forzature formali del verificazionismo non sembrano affatto sostenibili, dall'altro, Putnam non cede né all' "estremo popperiano," in cui l'impossibilità di formulare teorie attraverso l'accumulazione di esperienze percettive si traduce in una reinterpretazione astratta dell'attività scientifica. D'altra parte non cede nemmeno all' "estremo kuhniano" in cui la cumulatività del sapere scientifico è addirittura bandita di principio dalla "tesi dell'incommensurabilità." A fronte di questi estremi, Putnam ripropone invece le istanze pragmatiste del "senso comune critico" peirceano, ispirate alla ragionevolezza e al buon senso. In particolare, a Popper, che nega che l'accumulazione di esperienze percettive possa portare a formulare teorie, Putnam risponde:

egli ha ragione nel sostenere che ciò non accada in senso meccanico o algoritmico, tale accumulazione porta però a formulare teorie nel senso che rappresenta una regolarità dotata di significato metodologico il fatto che (1) la mancanza di esperienza dei fenomeni e della conoscenza già acquisita dei fenomeni stessi diminuisce in modo notevole la probabilità di idee corrette; e che (2) un'esperienza molto vasta accresce in modo notevole la probabilità di idee corrette, o parzialmente corrette. (Ibid.: 282; corsivo mio) ${ }^{25}$

Nei confronti di Kuhn, per il quale l'intervento di fattori extra-metodologici nella giustificazione scientifica sancirebbe la radicale storicizzazione della conoscenza e la conseguente relativizzazione della verità, potrebbero invece risultare efficaci alcune considerazioni che Putnam adduce riguardo al carattere "ad-hoc" delle asserzioni 
ausiliarie, le quali, essendo proprio ipotesi sulle condizioni iniziali e al contorno, rappresenterebbero l'emblema di quella "extra-metodologicità" che, secondo Kuhn, costringerebbe l'impresa scientifica ad abdicare dalla pretesa di razionalità.

Putnam osserva innanzitutto come la connotazione usuale di "arbitrarietà," e quindi di "irragionevolezza," che viene attribuita all'espressione "ad hoc" sia fuorviante:

"ad hoc" significa soltanto "per questo scopo particolare." È poi vero che "ad hoc" ha acquisito la connotazione di "irragionevole" - ma questa è una cosa diversa. L'ipotesi che certe stelle abbiano compagne oscure è "ad hoc" nel senso letterale: l'ipotesi viene formulata allo scopo specifico di rendere conto del fatto che non è visibile alcuna compagna. Ed essa è anche molto ragionevole. (Ibid.: 290)

D'altra parte, è inoltre sempre possibile verificare che l'intervento sulle asserzioni ausiliarie non sia arbitrario poiché, sebbene una teoria possa essere legittimamente preservata ricorrendo a mutamenti delle AA che siano ad hoc - ovviamente nel senso ragionevole appena proposto - i suoi successi non devono e non possono essere "ad hoc" - nel senso negativo dell'espressione; una teoria, infatti, viene accettata soltanto se è accompagnata da successi esplicativi sostanziali e reali, non "ad hoc." In entrambi gli argomenti Putnam fa ancora leva sul primato della prassi, ovvero sul successo esplicativo e applicativo delle teorie scientifiche come principale indizio della loro verità. Ma come possiamo finalmente giustificare in maniera apodittica questa tesi?

In genere, e a lungo andare, le idee vere sono quelle che hanno successo; come facciamo a saperlo? Anche questa asserzione è un'asserzione sul mondo, una asserzione alla quale siamo giunti in base alla nostra esperienza del mondo; e noi crediamo nella prassi a cui questa idea corrisponde, e nell'idea in quanto guida di quella prassi, perché crediamo in tutte le buone idee: tutto ciò ha dimostrato di avere successo! In questo senso "l'induzione è circolare." Ma certo che lo è! L'induzione non possiede alcuna giustificazione deduttiva; l'induzione non è deduzione. Le giustificazioni circolari non debbono essere necessariamente autoprotettive, né totalmente incapaci di fornire informazioni: i precedenti successi della "induzione" accrescono la fiducia che riponiamo in essa, e i suoi precedenti errori temperano tale fiducia. Il fatto che una giustificazione sia circolare significa solo che essa non può servire in se stessa come motivazione, a meno che la persona a cui viene fornita come motivazione non sia già in qualche modo propensa ad accettare la conclusione. Noi abbiamo una propensione - una propensione a priori, se volete - a ragionare in modo induttivo, e i precedenti successi della "induzione" accrescono tale propensione. Il metodo di controllare le idee attraverso la prassi e di basarsi su quelle che mostrano di avere successo (perché in ciò consiste l'induzione") non è ingiustificato. Si tratta di un'asserzione empirica. Il metodo non ha una "giustificazione" - se per giustificazione si intende una prova dedotta da princìi eterni e formali che giustifichi la fiducia nel metodo. Ma allora niente ha una giustificazione - nemmeno, a mio avviso, la matematica pura e la logica formale. (Ibid.: 293-4) ${ }^{26}$

Non esiste dunque, secondo Putnam, nessuna garanzia metodologica per instaurare una perfetta corrispondenza tra le nostre teorie alla realtà, ma ciò non significa che una tale relazione sia inconoscibile o che non esista. Il successo delle teorie e, più in generale, il progresso scientifico insegnano piuttosto che pensiero e realtà corrispondono "asintoticamente," in maniera graduale e approssimativa. L'epistemologo, assieme alle competenze tecniche e formali, dovrà allora esercitare altre virtù essenziali per il proprio mestiere: innanzitutto l'umiltà derivante dalla consapevolezza di non poter possedere metodologicamente la realtà, in secondo luogo la tenace fiducia nella possibilità della conoscenza per quanto incerta, relativa, provvisoria, ma comunque vera, cioè aperta al reale. 
Solo in questo modo sarà possibile liberarsi dai frustranti idoli del fondazionalismo riduzionistico, nonché dalla compiaciuta rassegnazione del relativismo: accettando con umiltà e fiducia la nostra condizione di animali razionali le cui idee - come abbiamo già detto - "non sono soltanto idee," ma lo strumento principale con cui modifichiamo il mondo per preservare la vita. Ecco allora che il primato della prassi quale criterio, o meglio, indizio nella lunga strada della verità offre alla conoscenza umana un fondamento dinamico ma allo stesso tempo ben radicato nel mondo della vita, che possiamo definire come la propensione a priori a ragionare in maniera induttiva, in virtù della fiducia riposta nelle esperienze che hanno mostrato di avere successo esplicativo e applicativo. Non occorre altra giustificazione.

La rilettura putnamiana dell'epistemologia contemporanea attraverso la lente del pragmatismo sembra, infine, essere ricca di insegnamenti anche per l'odierna coscienza culturale, testimoniando la possibilità di sintesi tra molteplici e differenti istanze filosofiche, riscoprendo il legame tra speculazione e vita con una rinnovata consapevolezza dell'attualità del sapere filosofico, valorizzando la complessa unità dell'umano, in cui ragione e emozioni contribuiscono insieme alla comprensione della realtà.

\section{BIBLIOGRAFIA}

ALTHUSSER Louis, (1965), Lire le Capital, Paris, Maspero; trad. it. ALTHUSSER Louis, (1968), Leggere il

Capitale, Milano, Feltrinelli.

AUXIER Randall E., ANDERSON Douglas R. \& Lewis Edwin HAHN (eds), (2015), The Philosophy of Hilary

Putnam, Chicago (Ill.), Open Court.

CALCATERRA Maria Rosa, (2003), Pragmatismo. I valori dell'esperienza, Roma, Carocci.

CARNAP Rudolf, (1934), Logische Syntax der Sprache, Wien, Julius Springer; trad. it., CARNAP Rudolf, (1966), La sintassi logica del linguaggio, Milano, Silva.

CARNAP Rudolf, (1939), "The Foundations of Logic and Mathematics," International Encyclopedia of Unified Science, I (3), Chicago, The University of Chicago Press; trad. it., CARNAP Rudolf, (1956), Fondamenti di logica e di matematica, Torino, Paravia.

CARNAP Rudolf, (1955), "Testability and Meaning," in Feigl H. \& Brodbeck M. (eds), Readings in the Philosophy of Science, New York, Appleton-Century-Crofts, 47-92.

CLARK Peter \& Bob hale (eds), (1994), Reading Putnam, Cambridge (Mass.), Basil Blackwell. CORVI Roberta, (2017), Ritorno al pragmatismo. L'alternativa Rorty-Putnam, Milano, Mimesis. DEWEY John, (1949), Logica. Teoria dell'indagine, Torino, Einaudi. HANSON Norwood Russell, (1958), Patterns of Discovery, Cambridge, Cambridge University Press; trad. it. HANSON Norwood Russell, (1978), I modelli della scoperta scientifica, Milano, Feltrinelli. JAMES William, (1879), “The Sentiment of Rationality,” Mind, IV (15) (Jul.), 317-46. 
JAMES William, (1912), The Will to Believe and Other Essays in Popular Philosophy, Auckland, Floating Press; trad. it. JAMES William, (1912), La volontà di credere, Milano, Libr. Ed. Milanese.

JAMES William, (1978), Pragmatism and The Meaning of Truth, Cambridge, Harvard University Press.

JAMES William, (2004), Principi di psicologia, Milano, Principato.

JAMES William, (2009), Saggi di empirismo radicale, Macerata, Quodlibet.

KUHN Thomas S., (1962), "The Structure of Scientific Revolutions," International Encyclopedia of Unified Science, II (2), Chicago, University of Chicago Press; trad. it. KUHN Thomas S., (1969), La struttura delle rivoluzioni scientifiche, Torino, Einaudi.

LIZZADRI Antonio, (2017), "Realismo scientifico senza scientismo: la proposta di Hilary Putnam," in D’Ariano G. M., Mariani C. \& Veca S. (a cura di), Realtà senza Realismo. Incontri di Studio n. 88, Milano, Istituto Lombardo - Accademia di Scienze e Lettere, 91-111.

MADDALENA Giovanni, (2009), Metafisica per assurdo. Peirce e i problemi dell'epistemologia contemporanea, Rubettino, Soveria Mannelli.

MARCHETTI Giancarlo, (2015), "Hilary Putnam," in Calcaterra R. M., Maddalena G. \& Marchetti G. (a cura di), Il pragmatismo. Dalle origini agli sviluppi contemporanei, Roma, Carocci, 289-306.

MISAK Cheryl J., (1991), Truth and the End of Inquiry, Oxford, Clarendon Press.

NIÑo Douglas, (2008), “Abducting Abduction. Avatares de la comprensión de la abducción de Charles S. Peirce" (Abducting Abduction. Vicissitudes in the Comprehension of Charles S. Peirce's Abduction), Ph.D. Thesis, Bogotá, Universidad Nacional de Colombia.

PASSMORE John, (1988), Recent Philosophers, La Salle, Open Court.

PEIRCE Charles Sanders, (1867), “On a New List of Categories," Proceedings of the American Academy of Arts and Sciences 7, 287-98.

PEIRCE Charles Sanders, (1877), “The Fixation of Belief,” Popular Science Monthly 12 (November), $1-15$.

PEIRCE Charles Sanders, (1878), "How to Make Our Ideas Clear," Popular Science Monthly 12 (January), 286-302.

PEIRCE Charles Sanders, (1891), “The Architecture of Theories,” The Monist, 1 (2) (January ), 161-76. PUTNAM Hilary, (1962), “What Theories Are Not,” in Nagel E., Suppes P. \& Tarski A. (eds), Logic, Methodology and Philosophy of Science, Stanford, Stanford University Press, 240-51; ripubblicato in PUTNAm Hilary, (1975), Mathematics, Matter and Method: Philosophical Papers, Volume I, Cambridge, Cambridge University Press, 215-27; trad. it. di G. Criscuolo, PUTNAM H., (1993), "Ciò che le teorie non sono," in Id., Matematica, materia e metodo, Milano, Adelphi, 237-50.

PUTNAM Hilary, (1973), "Explanation and Reference," in Pearce G. \& Maynard P. (eds), Conceptual Change, Dordrecht, D. Reidel Publishing Company, 199-221; ripubblicato in PUTNAM Hilary, (1975), Mind, Language and Reality: Philosophical Papers, Volume II, Cambridge \& New York, Cambridge University Press, 166-214; trad. it. di R. Cordeschi, PUTNAM Hilary, (2004 [terza edizione]), "Spiegazione e riferimento," in Id., Mente, linguaggio e realtà, Milano, Adelphi, 219-38. PUTNAm Hilary, (1974), “The ‘Corroboration' of Theories,” in Schlipp P. A. (ed.), The Philosophy of Karl Popper, La Salle (Ill.), The Open Court Publishing Co.; ripubblicato in Mathematics, Matter and Method: Philosophical Papers, Volume I, Cambridge, Cambridge University Press, 1975, 250-69; trad. 
it. di G. Criscuolo, PUTNAM Hilary, (1993), "La 'corroborazione' delle teorie," in Id., Matematica, materia e metodo, Milano, Adelphi, 273-94.

PUTNAM Hilary, (1975), “Language and Philosophy," in Id., Mind, Language and Reality: Philosophical Papers, Volume II, Cambridge \& New York, Cambridge University Press, 1-32; trad. it. di R. Cordeschi, PUTNAm Hilary, (2004 [terza edizione]), "Linguaggio e filosofia," in Id., Mente, linguaggio e realtà, Milano, Adelphi, 22-53.

PUTNAM Hilary, (1975b), “It Ain't Necessarily So," in Id., Mathematics, Matter and Method, Volume I, Cambridge, Cambridge University Press, 237-49 ; trad. it. PUTNAM Hilary, (1993), “Non è necessariamente così," in Id., Matematica, materia e metodo, Milano, Adelphi, 260-72.

PUTNAM Hilary, (1977), Realism and Reason, in Proceedings of the American Philosophical Association, 50, 483-98; ripubblicato in PUTNAM Hilary, (1978), Meaning and the Moral Sciences, London \& Boston, Routledge \& Kegan Paul, 123-8; trad. it. PUTNAM H., Realismo e Ragione, in Id. (1982), Verità e etica, Milano, Il Saggiatore, 141-60.

PUTNAM Hilary, (1978), "Meaning and Knowledge," in Id., Meaning and the Moral Sciences, London, Routledge and Kegan Paul, 123-38; trad. it. PUTNAM Hilary, (1982), "Significato e conoscenza," in Id., Verità e etica, Milano, Il Saggiatore, 17-96.

PUTNAM Hilary, (1978b), "Reference and Understanding," in Id., Meaning and the Moral Sciences, London \& Boston, Routledge \& Kegan Paul, 97-119; trad. it. PUTNAM Hilary, (1982), "Riferimento e comprensione," in Id., Verità e etica, Milano, Il Saggiatore, 115-38.

PUTNAM Hilary, (1981), Reason, Truth, and History, Cambridge (Mass.), Cambridge University Press; trad. it. di A. N. Radicati di Brozolo, PUTNAM Hilary, (1985) (Salvatore Veca ed.), Ragione, verità e storia, Milano, il Saggiatore.

PUTNAM Hilary, (1990), Realism with a Human Face, edited and introduced by James Conant, Cambridge (Mass.)/London, Harvard University Press.

PUTNAM Hilary, (1992), Il pragmatismo: una questione aperta, trad. it. M. Dell'Utri, Roma-Bari, Laterza.

PUTNAM Hilary, (2002), The Collapse of the Fact/Value Dichotomy and Other Essays, Cambridge (Mass.)/ London, Harvard University Press.

PUTNAM Hilary, (2012), Philosophy in an Age of Science, ed. by De Caro M. \& Macarthur D., Cambridge (Mass.)/London, Harward University Press; trad. it. PUTNAm Hilary, (2012), La filosofia nell'età della scienza, a cura di De Caro M. \& Macarthur D., Bologna, Il Mulino.

\section{NOTE}

1. In questa direzione vanno anche due recenti e autorevoli studi italiani, concordi nel riconoscere una sensibilità pragmatista nella prima fase delle ricerche putnamiane pur legate alle questioni epistemologiche tipiche della sua formazione riconducibile alla filosofia analitica. Cf. Corvi 2017, in particolare 89-98; Marchetti 2015. Inoltre, sia Corvi sia Marchetti riconoscono la propensione di Putnam a voler essere considerato un filosofo "senza aggettivi," reputando i movimenti filosofici come strumenti provvisori per introdurre idee nuove e non come categorie imprescindibili, entro cui il filosofo deve necessariamente e stabilmente collocarsi. La definizione di Putnam come un neopragmatista tout court sembra dunque essere per certi versi problematica.

2. Putnam 1977; trad. it. 1982. 
3. Putnam 1981; trad. it. 1985.

4. In letteratura critica (cf. Clark \& Hale 1994; Auxier, Anderson \& Hahn 2015) non vi sono evidenti ed esplicite interpretazioni neopositiviste della fase iniziale delle ricerche di Putnam, tuttavia, l'aver interpretato tali ricerche sempre e solo all'interno della tradizione e dello stile analitici, senza intravederne gli elementi di originalità, in primis, d'ispirazione pragmatista, ha indotto nell'immaginario filosofico l'idea di una fase neopositivista del pensiero di Putnam. È lo stesso Putnam ad aver denunciato in una delle ultime pubblicazioni l'indebita interpretazione neopositivista dei suoi primi scritti: “Il 'realismo scientifico', termine che avevo impiegato ripetutamente nei primi due volumi dei miei Philosophical Papers (apparsi nel 1975), viene identificato con quello che in Realismo e ragione avevo chiamato 'realismo metafisico': col risultato che si interpreta quella conferenza, e di fatto l'intero 'Putnam intermedio,' come una sconfessione di quasi tutto quello che avevo scritto nei due volumi retrospettivi [...] Ora si tende a credere che quei volumi rappresentino un periodo di 'realismo metafisico' nel corso della mia evoluzione! Per quanto un fraintendimento di questo genere sembri destinato a non scomparire dalla critica, permettetemi comunque di ripeterlo e sottolinearlo: mi sono sempre considerato un realista scientifico, anche se naturalmente non solo un realista scientifico." (Putnam 2012; trad. it. 2012, 74-5). D'altra parte, già ai tempi di Realism and Reason Putnam era consapevole del rischio di un simile fraintendimento: "Il 'realismo' di cui sto parlando viene spesso chiamato dai suoi sostenitori 'realismo scientifico.' Se evito qui tale termine [dopo averlo 'impiegato ripetutamente nei primi due volumi dei Philosophical Papers'], è perché 'realista scientifico,' come etichetta, comporta un certo tono ideologico - un tono reminiscente da vicino del materialismo del diciannovesimo secolo, o, per essere espliciti, dell'ateismo rozzo. In realtà, se un 'realista scientifico' è uno il quale crede, inter alia, che tutta la conoscenza degna del nome sia parte della 'scienza,' allora io non sono un 'realista scientifico.' Ma la conoscenza scientifica è certamente una fetta ragguardevole della nostra conoscenza; e della sua natura e del suo significato si sono occupati tutti i grandi filosofi minimamente interessati alla teoria della conoscenza [...] E se in ciò che segue concentrerò la mia attenzione sulla conoscenza scientifica, è perché su di essa si è concentrato il dibattito, e non per una mia personale propensione con lo scientismo." (Putnam 1978, 123-38; trad. it. 1982, 17-96). Per un approfondimento sul significato di "realismo metafisico" e "realismo scientifico" in Putnam e sulle possibili origini della loro confusione si veda Lizzadri (2017; in particolare 91-5). Si veda anche Marchetti (2015: 294): "Solitamente, gli interpreti del pensiero putnamiano sostengono che agli inizi della sua carriera egli abbia aderito al realismo metafisico. Questa affermazione non ci sembra del tutto corretta, poiché su di essa sembrano gravare talune 'sviste' interpretative. Sebbene in quegli anni egli abbia sostenuto un 'realismo privo di aggettivo qualificativo,' ci sembra inappropriato dire che egli, nella prima fase del suo pensiero, sia stato un realista metafisico. Infatti, le tesi sostenute in The Analytic and the Synthetic e What Theories Are Not mostrano come Putnam propenda per un realismo dalla connotazione scientifica."

5. Sempre riguardo al solipsismo metodologico del verificazionismo l'edizione in lingua originale propone un saggio non tradotto nell'edizione italiana. Si veda Putnam "Wittgenstein and Realism," in 2012: cap. 22.

6. Carnap 1934.

7. Putnam 1975; trad. it. 2004: 38.

8. La forza del linguaggio di dati sensoriali, che avrebbe dovuto spazzare via le impurità del linguaggio ordinario, risiede nella semplicità argomentativa con cui è possibile derivare le comuni esperienze percettive. Ad esempio, per raggiungere la conclusione formulata nel linguaggio di dati sensoriali "che nel mio campo visivo ho un dato sensoriale a forma di sedia," il ragionamento suonerebbe così: "(1) Sono in una stanza in cui si trova una sedia, e i miei occhi fissano la sedia. (2) La stanza è normalmente 
illuminata. (3) Io sono cosciente e presto attenzione al mio campo visivo. (4) Ogni volta che sono in una stanza in cui..., e che normalmente illuminata..., e io sono cosciente e presto attenzione al mio campo visivo, si dà il caso (o si dà quasi sempre il caso) che nel mio campo visivo c'è un dato sensoriale a forma di sedia. (Conclusione) Nel mio campo visivo c'è un dato sensoriale a forma di sedia. Il ragionamento mostra che la conclusione è derivabile dall'ultima premessa, che è una proposizione generale sulla percezione degli oggetti materiali ampiamente accettata, congiunta con altre tre premesse che sono 'ipotesi ausiliarie' alle quali io credo in questo caso specifico. Pertanto, tutto il mio sistema di credenze sulle cose materiali (e sulle loro relazioni con l'esperienza sensoriale) può spiegare (in sintonia con uno schema di spiegazione ampiamente esemplificato nella letteratura scientifica) un fatto indubbio circa la mia esperienza sensoriale. Se questo 'fatto indubbio' sia, in qualche senso, 'indubitabile,' e se sia effettivamente più 'basilare' dei fatti relativi a cose materiali [corsivo mio] non ha neppure bisogno di essere discusso. Tutto ciò che conta è che sia un fatto che l'avversario del realismo (ad esempio l'idealista soggettivista) sia disposto a riconoscere, e quindi un fatto che si possa invocare senza incorrere in una petitio principii." (Putnam 1975; trad. it. 2004: 42-3).

9. Come abbiamo appena constatato il giudizio di Putnam sul fenomenismo è categorico, tuttavia, ciò non gli impedisce di valorizzare il significato culturale dell'impresa neopositivista. Può essere utile acquisire famigliarità con tale propensione virtuosa caratteristica del pensiero di Putnam, per saperne riconoscere viziose strumentalizzazioni a favore dell'interpretazione neopositivista dei Philosophical Papers. Sempre riguardo al neopositivismo Putnam afferma: "L'importanza del contributo dell'empirismo logico è oggi spesso misconosciuta (o peggio, deliberatamente sminuita) perché il suo contributo effettivo si è rivelato interamente negativo. Non una delle grandi tesi positive dell'empirismo logico (che il significato è il metodo della verificazione; che le proposizioni metafisiche sono letteralmente prive di senso; che la matematica è vera per convenzione) è risultata valida. Certo, se $\mathrm{i}$ risultati che si ottengono sono uniformemente negativi, il fatto di poter rendere più scientifica la filosofia volgendo tesi filosofiche in tesi linguistiche e di poter stabilire con un'impegnata ricerca scientifica il valore di verità di certe proposizioni filosofiche perde un po' del suo potere di entusiasmo. Ma se la sensazione di disappunto è, umanamente parlando, del tutto comprensibile, non meno del desiderio di tentare qualcosa di nuovo, l'aver mostrato in che modo rendere più precise delle proposizioni filosofiche resta pur sempre un grande contributo storico. E se poi quelle proposizioni risultano tutte false, ebbene, aver raggiunto un accordo anche su ciò è certo un progresso importante." (Ibid.: 41).

10. Cf. Putnam 1973; trad. it. 2004: 232.

11. Putnam 1962; trad. it. 1993, in particolare: 237.

12. (Ibid.: 242).

13. La monografia di Carnap a cui si fa riferimento in questo passo è "The Foundations of Logic and Mathematics," (1939; trad. it. 1956).

14. Carnap 1955. Pubblicato in precedenza in Philosophy of Science, 3 (1936) e 4 (1937).

15. Peirce 1867. Sulle prime formulazioni della "massima pragmatista" si veda anche Peirce 1877, e Peirce 1878.

16. Tali questioni riguardanti l'interdipendenza tra osservazione e teoria, fatti e interpretazioni/ valori, ecc. verranno esplicitamente approfondite alla luce della tradizione pragmatista in Realism with a Human Face (1990), ne Il pragmatismo: una questione aperta (1992), per essere ancora riprese in The Collapse of the Fact/Value Dichotomy and Other Essays (2002). Al riguardo, il riferimento pragmatista principale è l'olismo di William James derivante dal suo empirismo radicale, secondo cui nell'"esperienza pura pensiero e sensazione sono fusi." Cf. James 2004; 2009. 
17. A questo riguardo, emerge come il pragmatismo costituisca un criterio interpretativo unitario del pensiero di Putnam: già nel 1962 - anno di pubblicazione del saggio in esame Putnam sosteneva l'esigenza di concretizzare la teoria del significato nella prassi linguistica, rendendola complementare ad una teoria della comprensione. Tale intuizione verrà tematizzata esplicitamente sedici anni dopo nel "manifesto" del realismo interno (Putnam 1978b; trad. it. 1982).

18. Putnam conia queste espressioni rispettivamente in Putnam 1974, trad. it. 1993; e in Putnam 1981; trad. it. 1985.

19. La riforma della teoria della verità come corrispondenza in senso dinamico come approssimazione asintotica tra pensiero e realtà viene esplicitamente proposta da Putnam in un altro saggio, pubblicato per la prima volta nei Philosophical Papers: "linguaggio e pensiero corrispondono asintoticamente alla realtà [...] Una teoria del riferimento è una teoria di tale corrispondenza." (Putnam 1975; trad. it. 2004: 317). Anche a questo proposito risulta evidente il rapporto con il pragmatismo: nella tradizione filosofica americana, la definizione di verità in termini di corrispondenza asintotica compare originariamente nei primi scritti di Peirce tra il 1860 e il 1870. L'immagine dell'asintoto, e dunque di una mai piena coincidenza tra piano del pensiero e piano della realtà, è segno di uno scetticismo positivo, che non vuole negare la possibilità della conoscenza, ma affermare l'indisponibilità almeno parziale della realtà da parte del soggetto conoscente. Nella prospettiva pragmatista, una teoria della conoscenza dovrà dunque rifuggire da tutti gli approcci alla realtà "totalizzanti," in cui il soggetto conoscente pretende possedere metodologicamente il reale, come avviene per il verificazionismo. Negli scritti dell'ultimo decennio del XIX secolo (si veda ad esempio Peirce 1891), Peirce sostiene che la logica dovrà piuttosto aprirsi ad altre scienze normative, quali l'estetica e l'etica, in quanto i segni non vengono interpretati attraverso una funzione logico-matematica, ma secondo una famigliarità estetica con il sistema di segni a cui appartengono, nonché secondo un giudizio etico sulla plausibilità di tale interpretazione. Non è evidentemente possibile formalizzare un simile processo (l'abduzione), che al contrario, secondo Peirce, potrà essere giustificato solo in virtù dell'“istinto razionale" degli esseri umani a interpretare i segni in maniera funzionale alla sopravvivenza. Come vedremo, anche in Putnam sarà riscontrabile un analogo ripensamento dell'induzione in termini di "propensione a priori" dell'intelligenza umana.

20. Circa il fondamento etico ed estetico della logica in Peirce si rimanda ai riferimenti bibliografici notati in precedenza (Supra, nota 19). Circa il rilievo cognitivo delle credenze morali e religiose si segnalano in particolare gli studi di un altro importante padre del pragmatismo: James 1912, trad. it. 1912; e James 1879.

21. Il teorema di Bayes asserisce, all'incirca, che la probabilità di un'ipotesi I data l'evidenza E è direttamente proporzionale alla probabilità di E data l'ipotesi I, ed è anche direttamente proporzionale alla probabilità antecedente di I - cioè alla probabilità di I allorché non si conosce E. Il teorema asserisce anche che la probabilità di I data l'evidenza E è minore, a parità di tutte le altre condizioni, se la probabilità di E sulla base dell'ipotesi I (non-I) è maggiore. Al giorno d'oggi i teorici della probabilità si dividono fra coloro che accettano la nozione di "probabilità antecedente di un'ipotesi," che è cruciale per il teorema, e coloro che respingono questa nozione, e quindi la nozione della probabilità di un'ipotesi sulla base di un'evidenza determinata. Quelli della prima scuola vengono chiamati "bayesiani"; gli altri "anti-bayesiani."

22. Anche rispetto al primato della prassi si riscontrano affinità con il pragmatismo classico: anche in Putnam, infatti, tale primato non può essere frainteso con una forma di utilitarismo soggettivistico, come è invece spesso erroneamente accaduto con l'interpretazione della teoria della verità in termini di "convenienza" proposta da William James (Cf. James 1978: 106; e, sulla letteratura critica, cf. Misak 1991; Calcaterra 2003). Putnam mette bene in luce come il primato della prassi quale criterio di giustificazione della verità di una teoria non debba essere inteso in termini egoistici ed utilitaristici, bensì in una prospettiva di convenienza collettiva derivante $\mathrm{e}$ 
ancorata al mondo della vita. In questa connessione tra esercizio della scienza, responsabilità pubblica e difesa della vita emerge un ulteriore riferimento ad un tema classico deweyano (cf. Dewey 1949).

23. I riferimenti bibliografici indicati sono: Hanson 1958, trad. it. 1978; Althusser 1965, trad. it. 1968; Kuhn 1962, trad. it. 1969.

24. Il saggio poi confluirà nel primo volume dei Philosophical Papers: Putnam 1975; trad. it. 1993. 25. È opportuno segnalare anche a questo riguardo la continuità rispetto alla tradizione pragmatista: già Peirce infatti aveva proposto una concezione probabilistica della conoscenza, opponendosi al necessitarismo della filosofia moderna e del positivismo (si veda sempre Peirce 1891). Secondo Peirce, l'uniformità in natura è solo statistica in quanto nella sua descrizione intervengono inevitabilmente fattori pratici che spezzano il legalismo a favore di un'immagine teleologica del cosmo, che, nella sua progressiva realizzazione delle condizioni migliori alla vita umana Peirce indica con il termine "agapismo." A sua volta, Putnam avrà modo di sviluppare ulteriormente l'argomento con considerazioni analoghe in Reason, Truth, and History, affermando che la maggiore o minore probabilità della verità di una descrizione, ovvero la sua accettabilità razionale, "vada ricercata nel fatto che avere un sistema di rappresentazioni di [un certo] tipo è parte della nostra idea di fioritura cognitiva umana, e perciò anche parte della nostra idea di fioritura umana totale, di eudemonia" (1981: 146).

26. Circa la "propensione a priori a ragionare in maniera induttiva" e dunque rispetto ad una conoscenza empirica che sia orientata e guidata da qualche ipotesi antecedente l'esperienza è possibile riscontrare le affinità con un tema classico del pragmatismo: l'abduzione. Sebbene nessun pragmatista mancò di occuparsi di abduzione in certa misura, è a Peirce che va attribuito il lavoro più dettagliato, ampio e approfondito su questo tipo di inferenza (per una ricostruzione approfondita, si veda Niño 2008; Maddalena 2009). Agli occhi di Peirce l'abduzione non rappresenta semplicemente quel momento interno alla pratica scientifica oggi noto come "inferenza alla migliore spiegazione," ma interessa anche il momento più propriamente ideativo o generativo in quanto tale, fino a costituire la struttura cognitiva del giudizio percettivo.

\section{RIASSUNTI}

The article aims at deepening and making explicit the influence of pragmatism on the thought of Hilary Putnam, moving from the analysis of some of his earlier writings, in which the reference to pragmatism was still very much implicit. In particular, although focused on the classical neopositivist questions of the philosophy of science on which Putnam has been trained, the essays What Theories Are Not (1962) and The "Corroboration" of Theories (1974) betray some original pragmatist options already. The article will review the pragmatist undertones in Putnam's criticism of contemporary epistemology in order to test its promises.

\section{AUTORE}

\section{ANTONIO LIZZADRI}

Università Cattolica del Sacro Cuore

Antonio.Lizzadri[at]unicatt.it 